\title{
An Efficient Replicated Data Management Approach for Peer-to-Peer Systems
}

\author{
J.H. Abawajy \\ Deakin University, \\ School of Information technology, \\ Geelong, Victoria, 3217 Australia
}

\begin{abstract}
The availability of critical services and their data can be significantly increased by replicating them on multiple systems connected with each other, even in the face of system and network failures. In some platforms such as peerto-peer (P2P) systems, their inherent characteristic mandates the employment of some form of replication to provide acceptable service to their users. However, the problem of how best to replicate data to build highly available peer-to-peer systems is still an open problem. In this paper, we propose an approach to address the data replication problem on $\mathrm{P} 2 \mathrm{P}$ systems. The proposed scheme is compared with other techniques and is shown to require less communication cost for an operation as well as provide higher degree of data availability.
\end{abstract}

\section{Introduction}

Peer-to-peer (P2P) network systems are one of the important and rapidly growing distributed system paradigms in which participants (the peers) rely on one another for service, rather than solely relying on dedicated and often centralized servers. The relationships among the nodes in the network are equal, nodes may join and leave the network in an ad-hoc manner and communication and exchange of information is performed directly between the participating peers. There is a growing research and industrial interest on peer-to-peer (P2P) systems. The success of P2P systems is due to many potential benefits such as fault-tolerance through massive replication; scaleup to very large numbers of peers, dynamic self-organization, load balancing, and parallel processing. Examples of P2P systems include Napster [3], Gnutella [4], and $\mathrm{KaZaA}[5]$ and Freenet [6].

Recently, peer-to-peer systems have become popular mechanism for large-scale content sharing. It is well known that techniques to increase the resilience and availability of stored data are fundamental to building dependable distributed systems. However, unlike traditional client-server applications that centralize the management of data in a few highly reliable servers, peer-to-peer systems distribute the burden of data storage, computation, communications and administration among thousands of individual nodes. Data management in this context offers new research opportunities since traditional distributed database techniques need to scale up while supporting high data autonomy, heterogeneity, and dynamicity. 
Generally P2P systems consider the data they offer to be very static or even readonly [9]. However, advanced peer-to-peer applications are likely to need more general replication capabilities. For example, a patient record may be replicated at several medical doctors and updated by any of them during a visit of the patient, e.g. to reflect the patient's new weight [8]. Other typical applications where new data items are added, deleted, or updated frequently by multiple users are bulletin-board systems, shared calendars or address books, e-commerce catalogues, and project management information [9]. Also, the inherent characteristics of peer-to-peer systems, require them to employ some form of replication to provide acceptable service to their users. For example, the erratic behaviour of online availability and the complete lack of global knowledge coupled with the absence of any centralisation makes P2P environments unreliable [9]. Ulike traditional distributed systems, the individual components of a peer-to-peer system experience an order of magnitude worse availability. This is because peer-to-peer systems are characterized by susceptibility to failure (e.g., node may be switched off), join and leave the system, have intermittent connectivity, and are constructed from low-cost low-reliability components. The study of a popular peer-to-peer file sharing system found that the majority of peers had availability rates of under $20 \%$ [1].

While much of the attention in the peer-to-peer systems research has been focused on the issues of providing scalability, free-rider problem or routing mechanisms within P2P networks, the resilience and availability of the data has so far seldom been mentioned. Therefore, how best to replicate data to build highly available peer-to-peer systems is still an open problem. In most peer-to-peer (P2P) systems data is assumed to be rather static and updates occur very infrequently. For application domains beyond mere file sharing such assumptions do not hold and updates in fact may occur frequently. Therefore, data replication in the presence of updates and transactions remains an open issue as well.

In this paper, we discuss an extension of our previous work on replica placement and management [2] to handle a system that support data replication in a transactional framework for weakly connected environments such as P2P systems. The proposed scheme uses quorum-based protocol for maintaining replicated data and shown to provide both high data availability and low response time. The proposed approach imposes a logical three dimensional grid structure on data objects based on a box shape organization and uses a sense-of-direction approach (SODA) for both read and write operations. We show that the proposed approach presents better average quorum size, high data availability, low bandwidth consumption, increased fault-tolerance and improved scalability of the overall system as compared to standard replica control protocols.

The rest of the paper is organized as follows. Section 2 presents related work. Section 3 presents our approach. Section 3 presents the proposed replica management protocol. In order to show the merits of the proposed approach, we present comparative analysis of the proposed approach against an existing approach in Section 4. Concluding remarks and future directions is reported in Section 5. 


\section{Related Work}

The rapid popularization of Internet-based P2P applications such as Napster [3], Gnutella [4], and KaZaA[5] has inspired the research and development of technologies for P2P services and systems. An efficient data replication management (DRM) technique is one of the important P2P technologies. Through an efficient DRM, the availability of $\mathrm{P} 2 \mathrm{P}$ services and their data can be significantly increased by replicating them on multiple systems connected with each other, even in the face of system and network failures [2]. From the viewpoint of data management these systems should address two critical areas [9]:

1. Efficient, scalable data access which is provided more or less by all approaches, and

2. Updates to the data stored, especially with respect to replication and low online probabilities.

However, the data sharing P2P systems like Gnutella and Kaaza deal with static, read-only files (e.g. music files) for which update is not an issue. Also, in systems such as Napster and Gnutella, replication occurs implicitly as each file downloaded by a user is implicitly replicated at the user's workstation. However, since these systems do not explicitly manage replication or mask failures, the availability of an object is fundamentally linked to its popularity and users have to repeatedly obtain the data. Also, if an update of a data item occurs this means that the peer that holds the item changes it. Subsequent requests would get the new version. However, updates are not propagated to other peers which replicate the item. As a result multiple versions under the same identifier (filename) may co-exist and it depends on the peer that a user contacts whether the latest version is accessed. The same holds true for most decentralised systems such as Gnutella [4].

ActiveXML [11] is a declarative framework that harnesses web services for data integration, and is put to work in a peer-to-peer architecture. It supports the definition of replicated XML fragments as Web service calls but does not address update propagation. Update is addressed in P-Grid [1], a structured network that supports selforganization. The update algorithm uses rumour spreading to scale and provides probabilistic guarantees for replica consistency. However, it only considers updates at the file level in a mono-master mode, i.e. only one (master) peer can update a file and changes are propagated to other (read-only) replicas.

Freenet [6] partially addresses updates which are propagated from the updating peer downward to close peers that are connected. Freenet uses a heuristic strategy to route updates to replicas which is uncertain to guarantee eventual consistency. Searches replicate data along query paths ("upstream"). In the case of an update (which can only be done by the data's owner) the update is routed "downstream" based on a key-closeness relation. Since the routing is heuristic, the network may change, and no precautions are taken to notify peers that come online after an update has occurred, consistency guarantees are limited. Also, peers that are disconnected do not get updated.

In OceanStore [10] every update creates a new version of the data object (versioning). Consistency is achieved by a two-tiered architecture: A client sends an update to the object's "inner ring" (some replicas who are the primary storage of the object and 
perform a Byzantine agreement protocol to achieve fault-tolerance and consistency) and some secondary replicas that are mere data caches in parallel. The inner ring commits the update and in parallel an epidemic algorithm distributes the tentative update among the secondary replicas. Once the update is committed, the inner ring multicasts the result of the update down the dissemination tree. To our knowledge analysis of the latency and consistency guarantees for this update scheme has not been published yet.

\section{Replica Management Approach}

Regardless of the underlying system topology, P2P systems need some form of replication to achieve good query latencies, load balance, and reliability. We now briefly describe the architecture of the proposed data replication system. Our system assumes an infrastructure-less peer-to-peer system, i.e., all peers are equal and no specialised infrastructure, e.g., hierarchy, exists. No peer has a global view of the system but base their behaviour on local knowledge, i.e., its routing tables, replica list, etc. The peers can go offline at any time according to a random process that models the behaviour when peers are online.

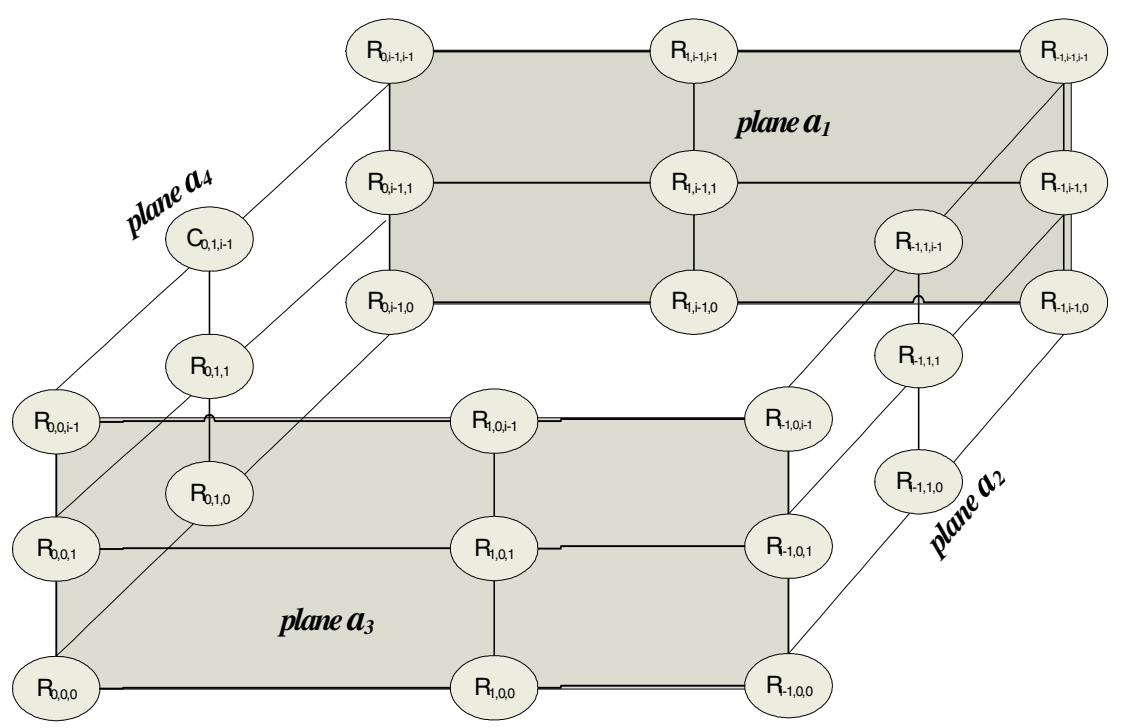

Fig. 1. The organization of replicas with four planes (i.e., $\alpha_{i}$ ) and the circles in the grid represent the sites

\subsection{System Architecture}

Given $\mathrm{N}$ copies of a data object, we logically organize the $\mathrm{N}$ copies into a box-shape structure with four planes (i.e., $\alpha_{1}, \alpha_{2}, \alpha_{3}$, and $\alpha_{4}$ ) as shown in Fig. 1. Each copy of the object (circles in Fig. 1) is located at $\mathrm{x}, \mathrm{y}, \mathrm{z}$ coordinate $\left(\mathrm{C}_{\mathrm{x}, \mathrm{y}, \mathrm{z}}\right)$ in a given plane 
(e.g., $\mathrm{C}_{0,0,0}, \mathrm{C}_{0,0,1}, \ldots, \mathrm{C}_{1-1,1-1,1-1}$ ). We define a pair of copies that can be constructed from a hypotenuse edge in a box-shape structure is called hypotenuse copies.

\subsection{Operations}

The basic architecture of Fig. 1 supports operations for creating objects, creating and deleting object replicas, and performing reads and writes on the shared objects in a transactional framework. Read operations on an object are executed by acquiring a read quorum that consists of any hypotenuse copies. In Fig. 1 , copies $\left\{R_{0,0,0}, R_{1-1,1-1,1-1}\right\}$, $\left\{\mathrm{R}_{0,0,1-1}, \mathrm{R}_{\mathrm{l}-1,1-1,0}\right\},\left\{\mathrm{R}_{0, \mathrm{l}-1,1-1}, \mathrm{R}_{\mathrm{l}-1,0,0}\right\}$, or $\left\{\mathrm{R}_{\mathrm{l}-1,0, \mathrm{l}-1}, \mathrm{R}_{0, \mathrm{l}-1,0}\right\}$ are hypotenuse copies and any one pair of which is sufficient to execute a read operation. Since each pair of them is hypotenuse copies, it is clear that, read operation can be executed if one of them is accessible, thus increasing the fault-tolerance of this protocol.

In contrast, write operations are executed by acquiring a write quorum from any plane that consists of: (1) hypotenuse copies; and (2) all vertices copies. For example, if the hypotenuse copies, say $\left\{\mathrm{R}_{0,0,0}, \mathrm{R}_{1-1,1-1,1-1}\right\}$ are required to execute a read operation, then copies $\left\{\mathrm{R}_{0,0,0}, \mathrm{R}_{1-1,1-1,1-1}, \mathrm{R}_{1-1,1-1,0}, \mathrm{R}_{0,1-1,1-1}, \mathrm{R}_{0,1-1,0}\right\}$ are sufficient to execute a write operation, since one possible set of copies of vertices that correspond to $\left\{R_{0,0,0}\right.$, $\left.\mathrm{R}_{1-1,1-1,1-1}\right\}$ is $\left\{\mathrm{R}_{1-1,1-1,1-1}, \mathrm{R}_{1-1,1-1,0}, \mathrm{R}_{0,1-1,1-1}, \mathrm{R}_{0,1-1,0}\right\}$. Other possible write quorums are $\left\{\mathrm{R}_{0,0,0}, \mathrm{R}_{\mathrm{l}-1,1-1,1-1}, \mathrm{R}_{\mathrm{l-1,1-1,0}, 0}, \mathrm{R}_{\mathrm{l}-1,0,1-1}, \mathrm{R}_{\mathrm{l}-1,0,0}\right\}, \quad\left\{\mathrm{R}_{\mathrm{l}-1,1-1,1-1}, \mathrm{R}_{0,0,0}, \mathrm{R}_{0,0,1-1}, \mathrm{R}_{\mathrm{l}-1,0,1-1}, \mathrm{R}_{\mathrm{l}-1,0,0}\right\}$, $\left\{\mathrm{R}_{\mathrm{l}-1,1-1,1-1}, \mathrm{R}_{0,0,0}, \mathrm{R}_{0,0,1-1}, \mathrm{R}_{0,1-1,1-1}, \mathrm{R}_{0,1-1,0}\right\}$, etc. It can be easily shown that a write quorum intersects with both read and write quorums in this protocol.

\subsection{Advantages}

The advantage of the proposed approach is that it tolerates the failure of more than three quarter of the copies. This is because the proposed protocol allows us to construct a write quorum even if three out of four planes are unavailable as long as the hypotenuse copies are accessible. To show this, consider the case when only one plane which consists of four copies of vertices and hypotenuse copies are available, e.g., the set $\left\{R_{1-1,1-1,1-1}, R_{0,0,0}, R_{0,0,1-1}, R_{1-1,0,1-1}, R_{1-1,0,0}\right\}$ is available as shown in Fig. 1. A transaction in the proposed can be executed successfully by accessing those copies in a quorum. Hence the write quorum in the proposed protocol is formed by accessing those available copies. Read operations, on the other hand, need to access the available hypotenuse copies.

Thus the proposed protocol enhances the fault-tolerance in write operations compared to the grid configuration protocol. Moreover, proposed protocol ensures that read operations have a significantly lower cost, i.e., two copies, and have a high degree of availability, since they are not vulnerable to the failure of more than three quarter of the copies. Write operations, on the other hand, are more available than the grid-based configuration protocol since only five copies are needed to execute write operations.

\section{Performance Analysis}

In the analysis of the update algorithm we focus on the amount of communication required to achieve consistency and provide probabilistic guarantees for successful 
and appropriate results for queries. To this end, we compared the proposed scheme with ROWA when the number of copies is set to 16 (i.e., $\mathrm{N}=16$ ). We also assumed that all copies have the same availability.

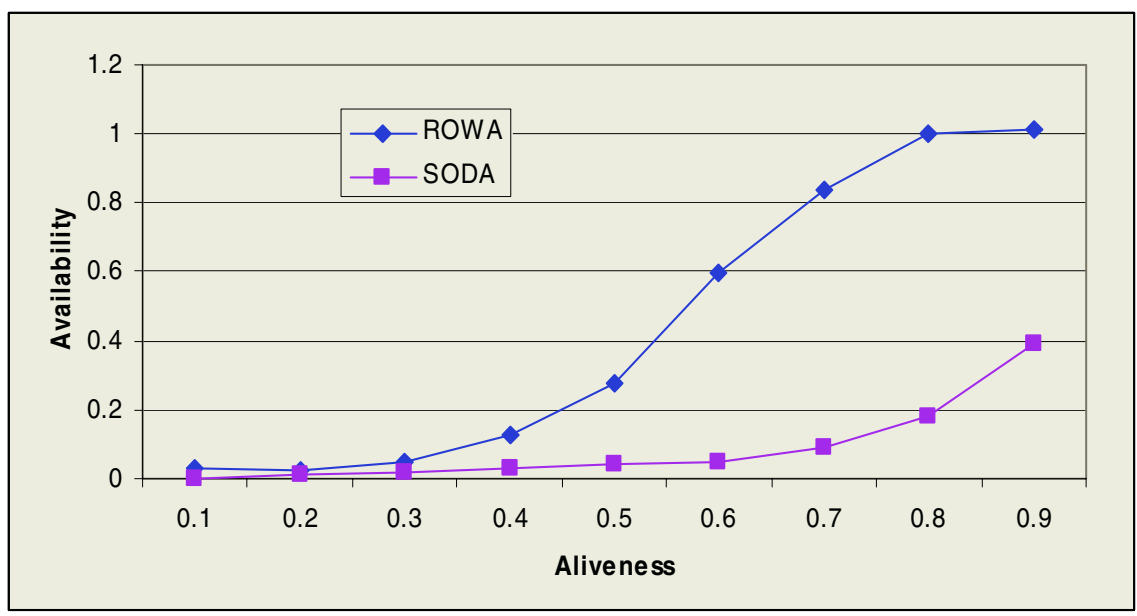

Fig. 2. Comparison of the write availability between SODA and ROWA

The result of the experiment is shown in Fig. 2. As shown, the proposed scheme has the lowest cost for write operation compared to ROWA protocol. This is because of the fact that in the ROWA protocol, an update operation needs to access all the replicas of the file in the system. Thus, the communication cost of an update operation in ROWA protocol is directly proportional to the number of replicas. In contrast, the proposed protocol needs only 5 copies at most, which results in significantly lower communication costs for comparable data availability.

\section{Conclusions and Future Directions}

The problem of protocol for maintaining replicated data has been widely studied in the distributed database systems. Recently, the need to support replication over widearea networks and to use the Internet infrastructure as the basis for building a perpetual data store has spawned new research directions. Until recently, the challenges in providing high availability to $\mathrm{P} 2 \mathrm{P}$ systems is poorly understood and only now being studied. Existing protocols are designed primarily to achieve high availability by updating a large fraction of the copies which provides some (although not significant) load sharing. We presented a new quorum-based protocol for maintaining replicated data across distributed P2P systems. The proposed approach is constructed on the organization of data in a box shape. We presented an analysis of the overhead and availability of the new protocol and showed that it performs better than the ROWA protocols. We are planning to implement the proposed protocol on various architectures including Data Grid, Peer-to-Peer, and Mobile systems. 
Acknowledgement. The help of Maliha Omar is greatly appreciated. Without her kind support, this paper would not have been completed. The financial support of Deakin University is also gratefully acknowledged.

\section{References}

1. S. Saroiu, P. K. Gummadi, and S. D. Gribble. A measurement study of peer-to-peer file sharing systems. In $M M C N, 2002$.

2. M. Mat Deris, J. Abawajy and H.M. Suzuri, "An Efficient Replicated Data Access Approach for Large-Scale Distributed Systems", In Proceedings of IEEE International Conference on Cluster and Grid Computing (CCGRID 2004).

3. Napster. http://www.napster.com/.

4. Gnutella. http://www.gnutella.com/.

5. KaZaA[KaZaA. http://www.kazaa.com/.

6. I. Clarke et al. Protecting Free Expression Online with Freenet. IEEE Internet Computing, 6(1), 2002.

7. Q. Lv, P. Cao, E. Cohen, K. Li, and S. Shenker. "Search and replication in unstructured peer-to-peer networks." In Proc. of the 16th annual ACM International Conf. on Supercomputing (ICS'02), New York, USA, June 2002.

8. R. Akbarinia, V. Martins, E. Pacitti, P. Valduriez.Replication and Query Processing in the APPA Data Management System, in: "Int. Workshop on Distributed Data and Structures (WDAS'2004), Lausanne", 2004.

9. Anwitaman Datta, Manfred Hauswirth, Karl Aberer,Updates in Highly Unreliable, Replicated Peer-to-Peer Systems,

10. S. Rhea, C. Wells, P. Eaton, D. Geels, B. Zhao, H. Weatherspoon, and J. Kubiatowicz. Maintenance-free global data storage. IEEE Internet Computing, 5(5), 2001.

11. Serge Abiteboul, Angela Bonifati, Gregory Cobena, Ioana Manolescu, Tova Milo: Dynamic XML Documents with Distribution and Replication, SIGMOD 2003 